This is a repository copy of A light clustering model predictive control approach to maximize termal power in solar parabolic-trough plants. in the Depósito de Investigación de la Universidad de Sevilla

Version: Author Accepted Version

Citation: Eva Masero, José Ramón D. Frejo, José M. Maestre, Eduardo F. Camacho. A light clustering model predictive control approach to maximize termal power in solar parabolic-trough plants. Solar Energy. Vol. 214. $10.1016 /$ j.solener.2020.11.056

To cite this publication, please use the final published version (if applicable). Please check the document version above.

Copyright: Other than for strictly personal use, it is not permitted to download, forward or distribute the text or part of it, without the consent of the author(s) and/or copyright holder(s), unless the work is under an open content license such as Creative Commons.

Takedown policy: Please contact us (idus@us.es) and provide details if you believe this document breaches copyrights. We will remove access to the work immediately and investigate your claim 


\title{
A light clustering model predictive control approach to maximize thermal power in solar parabolic-trough plants
}

\author{
Eva Masero ${ }^{\text {a,* }}$, José Ramón D. Frejo ${ }^{a}$, José M. Maestre ${ }^{\mathrm{a}}$, Eduardo F. Camacho ${ }^{\mathrm{a}}$ \\ ${ }^{a}$ Department of Systems and Automation Engineering, Universidad de Sevilla, C/ Camino de los Descubrimientos, s/n., Seville 41092, Spain.
}

\begin{abstract}
This article shows how coalitional model predictive control (MPC) can be used to maximize thermal power of large-scale solar parabolic-trough plants. This strategy dynamically generates clusters of loops of collectors according to a given criterion, thus dividing the plant into loosely coupled subsystems that are locally controlled by their corresponding loop valves to gain performance and speed up the computation of control inputs. The proposed strategy is assessed with decentralized and centralized MPC in two simulated solar parabolic-trough fields. Finally, results regarding scalability are also given using these case studies.
\end{abstract}

Keywords: Model predictive control, control by clustering, coalitional control, non-linear system, distributed solar collector field, thermal power.

\section{Introduction}

The global demand for energy and its adverse environmental impact significantly increased in the last decades due to population and economic growth. Therefore, finding an efficient, clean, and secure energy supply is one of the major challenges of the 21 st century. Renewable energy sources such as solar, wind, hydropower, biomass, and geothermal have been fostered as alternative and effective energy solutions (Twidell and Weir, 2015). Indeed, many researchers have focused on solar energy because it is the most abundant energy source (Camacho et al. 2012). The technologies most often used to convert solar energy into electrical power are photovoltaic cells (PV), which directly convert sunlight into electric energy, and concentrating solar power (CSP), which produces steam to drive turbine generators (Devabhaktuni et al., 2013). Regarding energy storage, CSP is preferred over PV due to the capacity of storing thermal energy so that electricity can be generated when there is low sunlight, e.g., on cloudy days or at night (Zhang et al., 2013).

In this work, we deal with the control of solar parabolictrough plants (Johansen and Storaa, 2002), one of the technologies within the CSP family, which also includes solar dishes (Santos-Martin et al., 2012), linear Fresnel collectors (Montes et al., 2014), and solar power towers (Salomé et al. 2013). From the control viewpoint, our challenge is to maximize power production despite the discontinuity of solar energy. Particularly, we consider valves at the beginning of all loops, which can be controlled to increase the resulting thermal power. Numerous control techniques have been proposed to this end, as shown in the comprehensive surveys of Camacho et al. (2007a b).

\footnotetext{
${ }^{*}$ Corresponding author

Email addresses: evamasero@us.es (Eva Masero), jdominguez3@us.es (José Ramón D. Frejo), pepemaestre@us .es (José M. Maestre), ef camacho@us.es (Eduardo F. Camacho)
}

One of the most relevant control strategies is model predictive control (MPC) (Rossiter. 2003), which is frequently used in the literature because it can handle challenging issues such as delay times, constraints, unstability, and nonminimum phase. An MPC application to a solar collector field is presented by Gallego and Camacho (2012), where an adaptive MPC controller using a Kalman filter to estimate temperature profiles and solar radiation is proposed. Moreover, Alsharkawi and Rossiter (2016) presented a dual MPC based on a linear estimated model for tracking and disturbance rejection. Nevertheless, nonlinear MPC (NMPC) is the most suitable control approach due to the behavior of the distributed parabolic-though field (Camacho and Gallego, 2015). For example, Gálvez-Carrillo et al. (2009) present an NMPC algorithm in combination with a Smith predictor to handle nonlinear dynamics and dead times, and Andrade et al. (2013) propose a robust NMPC that includes a Lyapunov function to guarantee stability despite uncertainties and measurements errors.

The majority of control approaches for solar collector fields focus on optimizing a global control input (i.e., the oil flow rate for the overall area) given set-points of the outlet temperature. Nevertheless, centralized strategies, as proposed by Johansen and Storaa (2002); Silva et al. (2003); Berenguel et al. (2005); Cirre et al. (2009); Torrico et al. (2010), can be impractical in large-scale systems due to the geographical spread and the system complexity. Distributed strategies have become suitable options to deal with these issues (Maestre and Negenborn, 2014). In such a way, the system is divided into subsystems controlled by cooperating local controllers, also known as agents, with partial information of the global system. There are various studies in this context, such as (Sánchez et al., 2018, Navas et al. 2018; Frejo and Camacho, 2020), where the control performance is analyzed when the inlet valves of each loop can be manipulated individually. 
A more recent control approach is that of clustering (Ding et al., 2016, Fele et al. 2017; Barreiro-Gomez et al., 2019, Ahandani et al., 2020), which allows dynamic cooperation between time-varying groups of local controllers. Indeed, system partitioning can be considered an offline clustering problem where subsystems are grouped most efficiently according to a given criterion. This idea can be generalized in a dynamic fashion so that the distributed control architecture can exploit the changing conditions of the system operation so as to decrease computation and cooperation burden with a minimal impact on performance. Furthermore, the partition of systems can be determined by a hierarchical or fully distributed control architecture. For example, La Bella et al. (2019) suggest a hierarchical approach for coordinating independent subsystems. In the lower layer, each agent solves a local MPC problem. In the upper layer, a supervisor checks and re-balances, if it is needed, the local control actions to guarantee global disturbance rejection. Tang et al. (2018) propose community detection as a tool to partition the system when using NMPC. Maestre and Ishii (2017) present a fully distributed method where the cooperation architecture is given by link requests/offers between local controllers; and in (Fele et al. 2018), agents perform an autonomous negotiation protocol to determine the partition of the system using coalitional MPC. Potential applications for clustering are power networks (Dörfler et al., 2014, Schiffer et al. 2017), freeways traffic systems (Chanfreut et al. 2020), wind farms (Yuan et al., 2018), telecommunication networks (Masero et al. 2020), irrigation canals (Fele et al., 2014), mobile social networks (Niyato et al., 2011), and supply chains (Gao et al. 2017). To the best of our knowledge, there are no applications in solar parabolic-trough plants.

This article proposes a hierarchical coalitional MPC strategy to reduce the computation burden and improve control performance in large-scale solar collectors fields. The twofold purpose is: i) maximizing the resulting thermal power, as studied before in the literature, and ii) promoting clusters of loops that directly optimize their control inputs according to a specific criterion. Finally, the proposed algorithm is simulated and assessed using two solar collectors fields. The first one is the ACUREX plant, a solar parabolic-trough plant of 10 loops at the Plataforma Solar de Almería (PSA) (Camacho et al., 2012, Gálvez-Carrillo et al., 2009), and the second one is a 100-loop parabolic-trough plant, which is an extension of the ACUREX field. These fields provide illustrative results of the scalability and performance of implementing the coalitional strategy compared to decentralized and centralized MPC methods. Under ideal conditions, with no clouds and collectors in perfect physical state, all MPC methods attain similar performance. The performance gain with coalitional control is mostly obtained when solar fields are unbalanced, i.e., when it presents large differences in its loop flow rates because clouds partially covering the field, and some collectors are dusty or in maintenance. This events combined with large-scale solar parabolic-trough plants become coalitional MPC into a potentially beneficial control strategy.
Index of contents. Section 2 introduces the model of a parabolic-trough plant. Section 3 explains the control objective and the coalitional MPC algorithm. Section 4 details the case studies and shows the assessment of coalitional MPC and other methods. Finally, conclusions are given in Section 5

\section{Parabolic-trough plant formulation}

This section presents a dynamic model of the solar field of a parabolic trough plant, i.e., a set of concave mirrors that concentrate the solar direct normal irradiance (DNI) on a tube located in its focal line. The heat transfer fluid (HTF) is circulated through the tube and absorbs the thermal energy to produce steam, which drives turbine generators to produce electricity using the power conversion system (PCS). Thermal energy storage (TES) can be employed to enhance the plant capacity for later use.

\subsection{System dynamics}

The collectors field can be modeled as a set of loops in parallel $\mathcal{J} \triangleq\left\{1, \ldots, N_{\text {loop }}\right\}$, which are divided into a set of segments $\mathcal{I} \triangleq\left\{1, \ldots, N_{\text {seg }}\right\}$ of length $\Delta l$ (see Fig. 11). Its dynamics is described by the following equations (Camacho et al. 2007a):

- the metal tube temperature in the segment $i$ of loop $j$ is

$$
\begin{aligned}
T_{i, j}^{\mathrm{m}}(k)= & T_{i, j}^{\mathrm{m}}(k-1)+\frac{\Delta t}{\rho^{\mathrm{m}} C^{\mathrm{m}} A^{\mathrm{m}}}\left(\eta_{i, j}^{\mathrm{col}} G_{i, j} I_{i, j}(k)\right. \\
& -\pi D^{\mathrm{m}} H_{i, j}^{\mathrm{l}}(k-1)\left(T_{i, j}^{\mathrm{m}}(k-1)-T^{\mathrm{a}}(k)\right) \\
& \left.-\pi D^{\mathrm{f}} H_{i, j}^{\mathrm{t}}(k-1)\left(T_{i, j}^{\mathrm{m}}(k-1)-T_{i, j}^{1 \mathrm{f}}(k-1)\right)\right),
\end{aligned}
$$

- and the HTF temperature in the segment $i$ of loop $j$ is

$$
\begin{aligned}
T_{i, j}^{\mathrm{f}}(k)=\quad & T_{i, j}^{\mathrm{f}}(k-1) \\
& +\frac{\pi D^{\mathrm{f}} H_{i, j}^{\mathrm{t}}(k-1) \Delta t}{\rho_{i, j}^{\mathrm{f}}(k-1) C_{i, j}^{\mathrm{f}}(k-1) A^{\mathrm{f}}}\left(T_{i, j}^{\mathrm{m}}(k-1)-T_{i, j}^{1 \mathrm{f}}(k-1)\right),
\end{aligned}
$$

with the auxiliary temperature $T_{i, j}^{1 \mathrm{f}}$ being

$$
T_{i, j}^{1 \mathrm{f}}(k)=T_{i, j}^{\mathrm{f}}(k)-\frac{q_{j}(k) \Delta t}{\Delta l A^{\mathrm{f}}}\left(T_{i, j}^{\mathrm{f}}(k)-T_{i-1, j}^{\mathrm{f}}(k)\right),
$$

where superscripts $\mathrm{m}, \mathrm{f}$, and a refer to the metal tube, the HTF, and the ambient respectively; $k$ is the discrete-time instant $t=k \Delta t ; i$ and $j$ denote, respectively, segment $i \in \mathcal{I}$ and loop $j \in \mathcal{J}$. The rest of model parameters and variables are given in Table 1 .

Observe that temperature $T_{i, j}^{\mathrm{f}}(k)$ is calculated assuming the fluid to be in a steady-state. Afterwards, the fluid temperature is corrected $\left(T_{i, j}^{1 \mathrm{ff}}(k)\right)$ using the net energy transported by the fluid. More information about the dynamics of the distributed parameter model can be found in (Camacho et al., 2012).

The temperature in the initial segments of loops is assumed to be equal to the inlet temperature $T_{1, j}^{\mathrm{f}}(k)=T^{\text {in }}(k)$ for all $k$ and $j \in \mathcal{J}$. Note that the inlet temperature $T^{\text {in }}(k)$, the corrected 


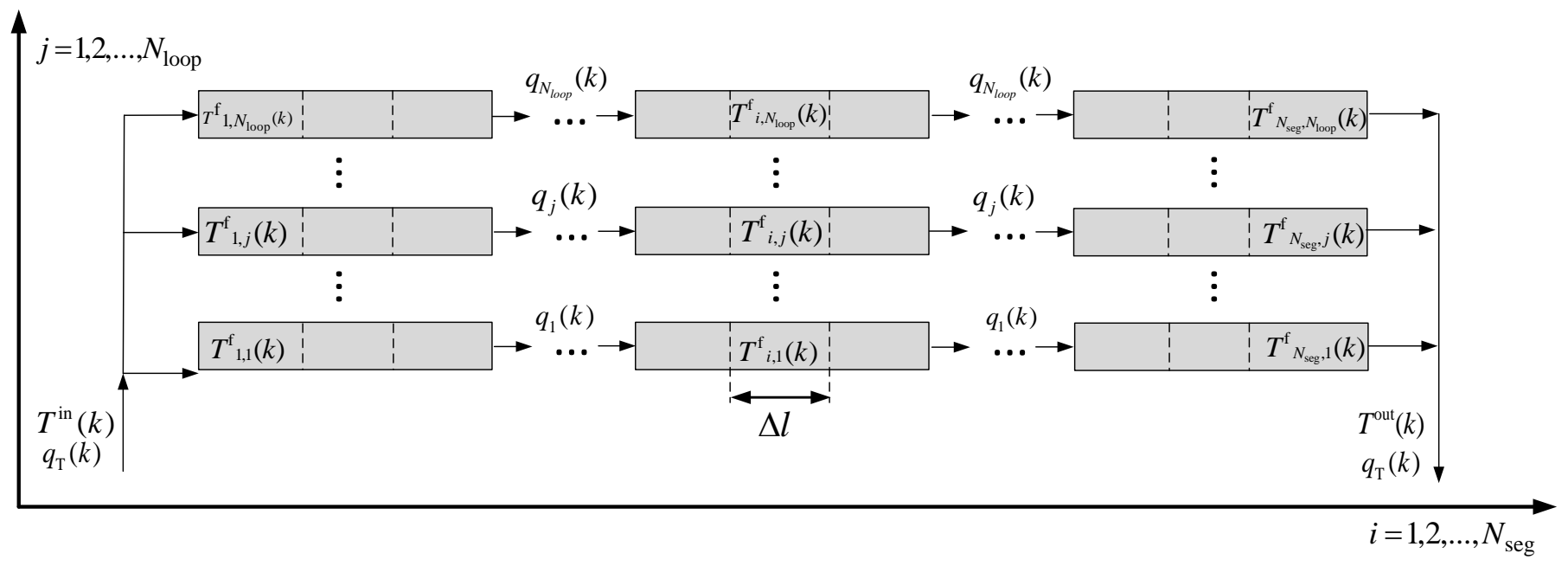

Figure 1: Schematic of the solar collector field.

Table 1: Model parameters and variables of the distributed collector field

\begin{tabular}{lll}
\hline Symbol & Description & Unit \\
\hline$\eta_{\text {col }}$ & Efficiency of collectors & - \\
$G$ & Aperture of collectors & $\mathrm{m}$ \\
$I$ & Direct solar irradiance & $\mathrm{W} / \mathrm{m}^{2}$ \\
$D^{\mathrm{m}}$ & Outside diameter of the pipe & $\mathrm{m}$ \\
$D^{\mathrm{f}}$ & Inside diameter of the pipe & $\mathrm{m}$ \\
$H^{\mathrm{t}}$ & Coef. of transmission metal-fluid & $\mathrm{W} /\left(\mathrm{m}^{2}{ }^{\circ} \mathrm{C}\right)$ \\
$H^{\mathrm{l}}$ & Coef. of thermal losses & $\mathrm{W} /\left(\mathrm{m}^{2}{ }^{\circ} \mathrm{C}\right)$ \\
$q$ & Flow rate & $1 / \mathrm{s}$ \\
$\Delta t$ & Discretization time of the model & $\mathrm{s}$ \\
$\Delta l$ & Length of segments & $\mathrm{m}$ \\
$\rho^{\mathrm{m}}, \rho^{\mathrm{f}}$ & Densities & $\mathrm{kg} / \mathrm{m}^{3}$ \\
$C^{\mathrm{m}}, C^{\mathrm{f}}$ & Specific heat capacities & $\mathrm{J} /\left(\mathrm{kg}{ }^{\circ} \mathrm{C}\right)$ \\
$A^{\mathrm{m}}, A^{\mathrm{f}}$ & Cross-sectional areas & $\mathrm{m}$ \\
$T^{\mathrm{m}}, T^{\mathrm{f}}, T^{\mathrm{a}}$ & Temperatures & ${ }^{\circ} \mathrm{C}$ \\
\hline
\end{tabular}

solar DNI $I_{i, j}(k)$, and the ambient temperature $T^{\mathrm{a}}(k)$ are considered as disturbances that can be measured or estimated at instant $k$ from the control viewpoint. Moreover, the outlet temperature of the field is calculated as

$$
T^{\text {out }}(k)=\frac{\sum_{j \in \mathcal{J}} q_{j}(k) T_{N_{\text {seg }}, j}^{\mathrm{f}}}{q_{\mathrm{T}}(k)},
$$

where the total flow rate is $q_{\mathrm{T}}(k)=\sum_{j \in \mathcal{J}} q_{j}(k)$.

\subsection{Operational constraints}

Several operational constraints must be taken into account. Firstly, constraints on the flow rate of each loop $j \in \mathcal{J}$ are considered as follows

$$
q_{j}^{\min } \leq q_{j}(k) \leq q_{j}^{\max },
$$

where $q_{j}^{\min }>0$ is the minimum value, which is based on the Reynolds number, and $q_{j}^{\max }>q_{j}^{\min }$ is the maximum flow rate related to the maximum pressure drop allowed. The total flow is also upper bounded by

$$
q_{\mathrm{T}}(k) \leq q_{\mathrm{T}}^{\max },
$$

where $q_{\mathrm{T}}^{\max }$ is the maximum total flow rate. Additionally, the outlet temperature of each loop $j \in \mathcal{J}$ is constrained as

$$
T^{\mathrm{f}, \min } \leq T_{N_{\mathrm{seg}}, j}^{\mathrm{f}}(k) \leq T^{\mathrm{f}, \max },
$$

with $T^{\mathrm{f}, \min }$ and $T^{\mathrm{f}, \max }$ being the minimum and the maximum temperature of the HTF, respectively. Note that constraints on the outlet temperature of each loop $T_{N_{\text {seg }}, j}^{\mathrm{f}}$ are established to hold the overall outlet temperature $T^{\text {out }}$ within its operational limits.

\section{Coalitional MPC algorithm}

Commercial plants usually operate at the maximum temperature to increase the efficiency of the power cycle and reduce the electrical consumption of the pumps. Conversely, thermal losses also increase, and thus, it does not necessarily imply an improvement in global efficiency, as discussed by Camacho and Gallego (2013). For that reason, the main objective of the coalitional MPC algorithm is to maximize thermal power resulting from the parabolic-trough plant while maintaining flow rates and the temperature of HTF below the maximum values despite disturbances such as solar irradiance changes due to clouds.

\subsection{Cooperative network and coalitions}

The parabolic-trough plant can be considered a cooperative network described by a graph $\mathcal{G}(k) \triangleq(\mathcal{J}, \mathcal{L}(k))$, where $\mathcal{J}$ is the set of loops, and $\mathcal{L}(k) \subseteq \mathcal{J} \times \mathcal{J}$ denotes the time-variant set of active links. Each loop $j \in \mathcal{J}$ is assumed to be managed by a local controller with local information from the plant, and each enabled link $l \in \mathcal{L}(k)$, which involves a cost $\varphi_{l} \in \mathbb{R}_{0+}$, establishes a bidirectional information flow between the corresponding loop controllers. 
Definition 1 (Coalition of loops). The graph $\mathcal{G}(k)$ defines a partition of the set of loops $\mathcal{J}$ into $\mathcal{P}(k) \triangleq\{1, \ldots, C(k)\}$ nonoverlapping, non-empty clusters of loops, referred to as coalitions, which satisfy $\cup_{\mathcal{C} \in \mathcal{P}(k)} C=\mathcal{J}$, i.e., the set of coalitions includes all loops.

Remark 1. By definition, the size of a coalition $C \in \mathcal{P}(k)$ can range from a single loop $C=\{j\}$ to the great coalition $C=\mathcal{J}$.

The outlet temperature of coalition $C \in \mathcal{P}(k)$ is

$$
T_{C}^{\mathrm{out}}(k)=\frac{\sum_{j \in C} q_{j}(k) T_{N_{\mathrm{seg}}, j}^{\mathrm{f}}}{q_{C}(k)},
$$

where the coalition flow rate is obtained by aggregating the flow rates of all loops grouped in $C$ as $q_{C}(k)=\sum_{j \in C} q_{j}(k)$, which is lower and upper bounded by $q_{C}^{\min }=\sum_{j \in C} q_{j}^{\min }$ and $q_{C}^{\max }=$ $\sum_{j \in C} q_{j}^{\max }$, respectively.

\subsection{Control objective}

Let $\mathcal{P}(k)$ be the partition of the plant at time instant $k$. Each coalition $C \in \mathcal{P}(k)$ is managed by a single controller, whose control goal is to minimize the cost function

$$
\begin{aligned}
J_{C}(\cdot)= & \sum_{n=1}^{N_{\mathrm{p}}}-W_{C}(k+n)+\alpha_{C} \Psi_{C}\left(T_{N_{\mathrm{seg}}, j}^{\mathrm{f}}(k+n)\right) \\
& +\beta_{C} \sum_{n=0}^{N_{\mathrm{p}}-1} \sum_{j \in C}\left(q_{j}(k+n)-q_{j}(k+n-1)\right)^{2},
\end{aligned}
$$

where $N_{\mathrm{p}}$ is the prediction horizon ${ }^{1} k+n$ denotes the prediction $n$ steps ahead at time instant $k$; and $W_{C}$ is the thermal power:

$$
\begin{aligned}
W_{C}(k+n) & =W_{C}^{\text {out }}(k+n)-W_{C}^{\text {in }}(k+n) \\
& =\sum_{j \in C} W_{j}^{\text {out }}(k+n)-\sum_{j \in C} W_{j}^{\text {in }}(k+n),
\end{aligned}
$$

with $W_{j}^{\text {out }}$ and $W_{j}^{\text {in }}$ respectively being

$$
\begin{aligned}
& W_{j}^{\text {out }}(k+n)=\rho_{N_{\text {seg }, j}}^{\mathrm{f}}\left(T_{N_{\text {seg }}, j}^{\mathrm{f}}(k+n)\right) C_{N_{\text {seg }}, j}^{\mathrm{f}}\left(T_{N_{\text {seg }}, j}^{\mathrm{f}}(k+n)\right) q_{j}(k+n) T_{N_{\text {sce }, j}}^{\mathrm{f}}(k+n), \\
& W_{j}^{\mathrm{in}}(k+n)=\rho_{1, j}^{\mathrm{f}}\left(T_{1, j}^{\mathrm{f}}(k+n)\right) C_{1, j}^{\mathrm{f}}\left(T_{1, j}^{\mathrm{f}}(k+n)\right) q_{j}(k+n) T_{1, j}^{\mathrm{f}}(k+n) .
\end{aligned}
$$

Here $W_{C}^{\text {out }}, W_{j}^{\text {out }}$ and $W_{C}^{\text {in }}, W_{j}^{\text {in }}$ are, respectively, the outlet and input thermal powers of coalition $C$ and loop $j ; \alpha_{C}, \beta_{C} \in \mathbb{R}_{>0}$ are tuning parameters; and $\Psi_{C}\left(T_{N_{\text {seg }}, j}^{\mathrm{f}}(k+n)\right)$ penalizes the maximum quadratic violation of the outlet temperature constraints of coalition $C$ :

$\Psi_{C}\left(T_{N_{\text {seg }}, j}^{\mathrm{f}}(k+n)\right)=\sum_{j \in C} \max \left(\frac{T_{N_{\text {seg }, j}}^{\mathrm{f}}(k+n)-T^{\mathrm{f}, \max }}{T^{\mathrm{f}, \max }}, \frac{T^{\mathrm{f}, \min }-T_{N_{\text {seg }, j}}^{\mathrm{f}}(k+n)}{T^{\mathrm{f}, \max }}, 0\right)^{2}$.

\footnotetext{
${ }^{1}$ The prediction horizon is sometimes used together with the so-called control horizon $N_{\mathrm{u}}$ to reduce the computation burden and account for delays, with $N_{\mathrm{u}}<N_{\mathrm{p}}$. The prediction horizon represents how many future time instants of the system evolution are included in the cost function. The control horizon stands for the number of actual time steps for which a different input needs to be computed, and the input is assumed to remain constant beyond $k+N_{\mathrm{u}}$.
}

Observe that (11), which corresponds to the second term of (9), is used as a soft constraint. Thus, given a large $\alpha_{C}$, the coalitional controller will strive to keep the outlet temperature within its bounds; otherwise, the constraint will be violated. Note that this soft constraint is employed to avoid unfeasibility issues when solving the optimization problem of the proposed algorithm. Moreover, the third term of (9) penalizes the variations in the input according to the value of $\beta_{C}$ so that the flow rate oscillation can be reduced in all the valves during daily operation.

Remark 2. The exact calculation of thermal power $W_{C}$ requires to include how its density and specific heat capacity vary with temperature along the corresponding segments.

\subsection{Control algorithm}

Taking into account the coupling between loops in the total flow $q_{\mathrm{T}}$, the collector field can be unbalanced, i.e., there can be large differences in local flow rates due to the solar DNI and the efficiency gaps between loops. Specifically, these gaps can appear when clouds partially cover the plant, and when some collectors are dusty or in maintenance.

To improve the performance in this situation, we propose a hierarchical coalitional MPC where the partition of the plant is determined by an upper control layer that associates unbalanced pairs of loops dynamically. Since the number of partitions combinatorially explodes with the cooperation links, and the coalitions size is related with computation time, we propose this clustering criterion to reduce options and find control solutions in real-time. Hence, loop $j_{\mathrm{a}}$ of coalition $C$ can benefit from the extra flow rate in loop $j_{\mathrm{b}} \in C \backslash\left\{j_{\mathrm{a}}\right\}$ so that the resulting thermal power can be increased. Specifically, loop $j_{\mathrm{b}}$ with lower solar DNI/efficiency can provide its extra flow rate to loop $j_{\mathrm{a}}$, which can squeeze its higher solar to obtain larger thermal power. This control strategy allows dealing with largescale plants, where the centralized solution is highly complex and impossible to be computed in real-time.

The proposed coalitional MPC algorithm is summarized in Algorithm 1.

\footnotetext{
Algorithm 1

Let us define the flow rate vector of $C$ as $Q_{C}(k) \triangleq$ $\left[q_{C}(k), q_{C}(k+1), \ldots, q_{C}\left(k+N_{\mathrm{u}}-1\right)\right]$ for all $C \in \mathcal{P}(k)$, with $N_{\mathrm{u}}$ being the control horizon, which is the number of inputs that needs to be computed by the optimizer, and the rest of control moves are held constant $\left(N_{\mathrm{u}} \leq N_{\mathrm{p}}\right)$.

At each time instant $k$ :
}

1. The upper control layer measures local flow rate $q_{j}(k-$ 1) for all loops $j \in \mathcal{J}$. Let us define a vector $Q_{\mathcal{J}}$, which contains the flow rate of all loops at the previous instant $k-1$ sorted in ascending/descending order, and let us also define the index $I_{\mathcal{J}}$ which specifies how the loops are rearranged to obtain the sorted vector $Q_{\mathcal{J}}$.

2. The upper layer computes the plant partition $\mathcal{P}(k)=$ $\{1,2, \ldots, C(k)\}$ associating pairs of unbalanced loops. 
Particularly, coalitions $C \in \mathcal{P}(k)$ are formed as

$$
C \triangleq\left\{I_{\mathcal{J}}(m), I_{\mathcal{J}}\left(N_{\text {loop }}+1-m\right)\right\}, \quad \forall m \in\left|I_{\mathcal{J}}\right| .
$$

Note that, as the total number of loops is pair in both simulation scenarios, only clusters of two loops are considered. Afterwards, the corresponding instructions are sent to the lower control layer.

3. In the lower layer, coalitions receive their estimated solar DNI in the prediction horizon $N_{\mathrm{p}}$, and measure $T_{i, j}^{\mathrm{f}}(k), \forall i \in \mathcal{I}$ of their loops $j \in C$. Since there is not a sensor in each segment, some temperatures $T_{i, j}^{\mathrm{f}}$ are estimated based on available sensors measurements.

4. Each $C \in \mathcal{P}(k)$ solves the optimization problem:

$$
Q_{C}^{*}(k)=\arg \min _{Q_{C}(k)} J_{C}(\cdot),
$$

subject to dynamics (1)-(3), constraints (5), (7), and

$$
q_{C}^{\max }=\frac{q_{\mathrm{T}}^{\max }|C|}{N_{\text {loop }}} .
$$

Note that, in 12, it is computed the optimal flow rate of each loop belonging to coalition $C$, considering that any loop $j_{\mathrm{a}}$ of coalition $C$ can benefit from the extra flow rate in loop $j_{\mathrm{b}} \in C \backslash\left\{j_{\mathrm{a}}\right\}$ and vice versa. The flow rate $q_{C}(k)$ can be composed by the sum of flow rates of all loops belonging the coalition, i.e. $q_{C}(k)=\sum_{j \in C} q_{j}(k)$. Furthermore, $q_{C}(k)$ is used as control input of coalition $C$ assuming that the hydraulic dynamics is significantly faster than the thermal dynamics.

5. Each coalition $C \in \mathcal{P}(k)$ applies the first element of its optimal input vector $Q_{C}^{*}(k)$.

6. Set $k \leftarrow k+1$ and go to Step 1 .

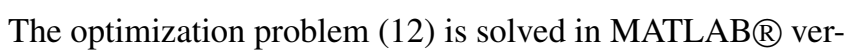
sion R2020a using the solver fmincon from the Optimization Toolbox with the algorithm option: interior-poin $2^{2}$ Additionally, the following simplifications are taken into account to decrease the computation burden: i) the coefficient of thermal losses $H^{1}$ is pre-computed for a given set of flow rates and temperatures, and ii) the sample time of the controller $\Delta t_{\text {ctr }}$ is selected as a multiple of the discretization time of the model $\Delta t$, i.e., $\Delta t_{\mathrm{ctr}}=\gamma \Delta t$ with $\gamma \in \mathbb{N}_{+}$.

Remark 3. This algorithm can be modified following other criteria to form coalitions, e.g., allowing clusters of different sizes or associating loops with high gaps of solar DNI or temperature. A further discussion is provided in subsection 4.3 .

Remark 4. If there were variables that cannot be measured, they could be estimated based on the rest of known variables,

\footnotetext{
${ }^{2}$ http://www.mathworks.com/help/optim/ug/constrained-nonlinearoptimization-algorithms.html\#brnpd5f
}

as performed with $T_{i, j}^{\mathrm{f}}(k)$. Particularly, the solar DNI along the prediction horizon can be estimated using several forecasting techniques (Law et al. 2014: Ramirez and Vindel, 2017) such as numerical weather prediction models (NWPM), time series analysis methods, cloud motion vectors (Alonso-Montesinos et al. 2015), and hybrid methods (Chu et al. 2013). Otherwise, the current solar DNI can be used, considering that the resulting thermal power can be reduced.

Furthermore, note that most commercial plants do not have flow meters in all loops $j \in \mathcal{J}$, but in many plants there are loop valves that can be controlled. Although flow rate $q_{j}(k)$ is not usually measured, it can be estimated from $q_{\mathrm{T}}(k)$, which is often measured, and the valve position of all loops with the aid of a hydraulic model. The temperatures of the loops can also be used for flow estimation.

\section{Illustrative examples}

This section details the 10-loop and 100-loop solar parabolictrough plants employed to test the proposed coalitional MPC algorithm. Apart from the number of loops, both plants have similar dynamics. Thus, the idea is to discuss the performance and scalability of the coalitional strategy in large-scale solar fields, compared with decentralized and centralized MPC methods.

\subsection{Case studies}

The first distributed solar collector field considered is the ACUREX plant, which was operating in the Plataforma Solar de Almería (PSA) (Gálvez-Carrillo et al., 2009). The electricity generation of ACUREX was made indirectly by concentrating solar power to produce the steam, which drives turbine generators to produce electricity using the PCS.

The solar field was composed of East-West aligned single axis parabolic-trough collectors. Specifically, there were $N_{\text {loop }}=10$ parallel loops of length $d_{\text {loop }}=174 \mathrm{~m}$, which were discretized into $N_{\text {seg }}=174$ segments of length $\Delta l=1 \mathrm{~m}$. Each loop $j \in\left\{1, \ldots, N_{\text {loop }}\right\}$ had twelve collectors connected in series, being the active part (that receiving solar irradiance) $144 \mathrm{~m}$ long, and the passive part $30 \mathrm{~m}$. Collectors consisted of linear parabolic mirrors that reflect and concentrate the solar power onto a tube located along the focal line. The HTF employed was the Therminol 55 thermal oil, whose density is $\rho_{i, j}^{\mathrm{f}}\left(T_{i, j}^{\mathrm{f}}(k)\right)$ and its specific heat capacity $C_{i, j}^{\mathrm{f}}\left(T_{i, j}^{\mathrm{f}}(k)\right)$ were computed as

$$
\begin{aligned}
& \rho_{i, j}^{\mathrm{f}}\left(T_{i, j}^{\mathrm{f}}(k)\right)=903-0.672 T_{i, j}^{\mathrm{f}}(k), \\
& C_{i, j}^{\mathrm{f}}\left(T_{i, j}^{\mathrm{f}}(k)\right)=1820+3.478 T_{i, j}^{\mathrm{f}}(k) .
\end{aligned}
$$

The metal-fluid transmission and thermal losses coefficients were, respectively, defined by

$$
\begin{aligned}
H_{i, j}^{\mathrm{t}}\left(q_{i}(k), T_{i, j}^{\mathrm{f}}(k)\right) & =q_{i}^{0.8}(k) \Phi\left(T_{i, j}^{\mathrm{f}}(k)\right), \\
H_{i, j}^{\mathrm{l}}\left(T_{i, j}^{\mathrm{f}}(k), T^{\mathrm{a}}(k)\right) & =0.00249\left(T_{i, j}^{\mathrm{f}}(k)-T^{\mathrm{a}}(k)\right)-0.06133,
\end{aligned}
$$

where

$$
\begin{aligned}
\Phi\left(T_{i, j}^{\mathrm{f}}(k)\right)= & 2.17 \cdot 10^{6}-5.01 \cdot 10^{4} T_{i, j}^{\mathrm{f}}(k)+4.53 \cdot 10^{2} T_{i, j}^{\mathrm{f}}(k)^{2} \\
& -1.64 T_{i, j}^{\mathrm{f}}(k)^{3}+2.1 \cdot 10^{-3} T_{i, j}^{\mathrm{f}}(k)^{4},
\end{aligned}
$$


following (Camacho et al., 2012). The rest of model parameters and constraints are displayed in Table 2. Note that the ambient temperature $T^{\mathrm{a}}(k)$ can be considered constant for simplicity.

Table 2: Model parameters and constraints of ACUREX plant

\begin{tabular}{lll}
\hline Symbol & Value & Unit \\
\hline$\rho^{\mathrm{m}}$ & 7800 & $\mathrm{~kg} / \mathrm{m}^{3}$ \\
$C^{\mathrm{m}}$ & 550 & $\mathrm{~J} /\left(\mathrm{kg}^{\circ} \mathrm{C}\right)$ \\
$A^{\mathrm{f}}$ & $7.55 \cdot 10^{-4}$ & $\mathrm{~m}^{2}$ \\
$D^{\mathrm{m}}$ & 0.031 & $\mathrm{~m}$ \\
$D^{\mathrm{f}}$ & 0.0254 & $\mathrm{~m}$ \\
$q_{j}^{\min }$ & 0.2 & $1 / \mathrm{s}$ \\
$q_{j}^{\max }$ & 1.5 & $1 / \mathrm{s}$ \\
$q_{\mathrm{T}}^{\max }$ & 6.5 & $1 / \mathrm{s}$ \\
$T^{\mathrm{a}}$ & 25 & ${ }^{\circ} \mathrm{C}$ \\
$T^{\mathrm{f}, \min }$ & 220 & ${ }^{\circ} \mathrm{C}$ \\
$T^{\mathrm{f}, \max }$ & 300 & ${ }^{\circ} \mathrm{C}$ \\
\hline
\end{tabular}

The inlet temperature of the field can be expressed as a firstorder system:

$$
\frac{T^{\text {in }}(s)}{\hat{T}^{\text {out }}(s)}=\frac{1}{600 s+1},
$$

where $\hat{T}^{\text {out }}(s)=T^{\text {out }}(s)-90$, with a time constant of $10 \mathrm{~min}$. Notice that a temperature drop of $90^{\circ} \mathrm{C}$ was considered in the steam generator. The discrete-time equation of the inlet temperature with discretization time model $\Delta t=0.5 \mathrm{~s}$ is

$$
T^{\text {in }}(k)=0.999167 T^{\text {in }}(k-1)+8.33 \cdot 10^{-4}\left(T^{\text {out }}(k-1)-90\right) .
$$

The second distributed collector field considered is formed by $N_{\text {loop }}=100$ loops. Since it is an extension of the ACUREX plant (10-loop plant), the previous equations are held. Constraints and model parameters are these of Table 2 except for the maximum total flow rate, which becomes $q_{\mathrm{T}}^{\max }=65 \mathrm{l} / \mathrm{s}$.

\subsection{Simulation results}

All optimization problems have been solved using MATLAB $\AA$ on Windows with a PC Intel $\AA$ Core $^{\mathrm{TM}}$ i7$8700 \mathrm{CPU}$ at $3.20 \mathrm{GHz}$ and $16 \mathrm{~GB}$ RAM. The prediction horizon is $N_{\mathrm{p}}=12$, the control horizon is $N_{\mathrm{u}}=10$, and the tuning parameters are $\alpha_{j}=45$ and $\beta_{j}=3$. N.B.: The horizons have been selected by trial and error to reach the maximum performance with reasonable computation effort. The value of $\alpha_{j}$ is set large enough to keep the outlet temperature within its bounds. Additionally, the value of $\beta_{j}$ has been appropriately selected to represent the valves control effort, considering that a small and large value will lead to more and less flow rate oscillation in all the valves during daily operation, respectively. Although the length of segments is $\Delta l=1 \mathrm{~m}$ and the discretization time is $\Delta t=0.5 \mathrm{~s}$ in the simulation model, the MPC controllers consider $\Delta l=6 \mathrm{~m}$ and $\Delta t=3 \mathrm{~s}$ for the model to speed up the computation time. Thus, the sample time of controllers is $\Delta t_{\mathrm{ctr}}=60 \mathrm{~s}$ by setting $\gamma=20$. Moreover, it is considered temperatures $T^{\text {in }}, T_{1, j}^{\mathrm{f}}, T_{1, j}^{1 \mathrm{f}}=155^{\circ} \mathrm{C}$ and $T_{1, j}^{\mathrm{m}}=165^{\circ} \mathrm{C}, \forall j \in \mathcal{J}$ at time instant $k=0$.
The average computation time per number of coalitions in a simulation of length $N_{\text {sim }}$ is computed as

$$
\tau_{C}=\frac{\tau_{\text {total }}}{\bar{C}}, \text { with } \bar{C}=\frac{\sum_{k=1}^{N_{\text {sim }}} \sum_{C \in \mathcal{P}(k)} C}{N_{\text {sim }}},
$$

to compare the following control strategies:

1. Local MPC presents a decentralized MPC where each loop $j \in \mathcal{J}$ is managed by a local controller with no information about the rest of loops, i.e., $C=\{j\}$ for all $C \in \mathcal{P}(k)$. Note that the maximum flow in each loop becomes $q_{j}^{\max }=q_{\mathrm{T}}^{\max } / N_{\text {loop }}$ in this case.

2. Cen MPC consists of a centralized MPC where the control problem is solved for the grand coalition $C=\mathcal{J}$.

3. No-valves refers to a centralized MPC method where it is assumed that valves cannot be controlled and, thus, the same flow rate is applied to each loop.

4. Coal MPC indicates the proposed coalitional MPC as detailed in Algorithm 1, which promotes clusters $C \triangleq$ $\left\{j_{1}, j_{2}\right\} \in \mathcal{P}(k)$ of two loops $j_{1}, j_{2} \in \mathcal{J}$ with high flow rate gaps to balance the field.

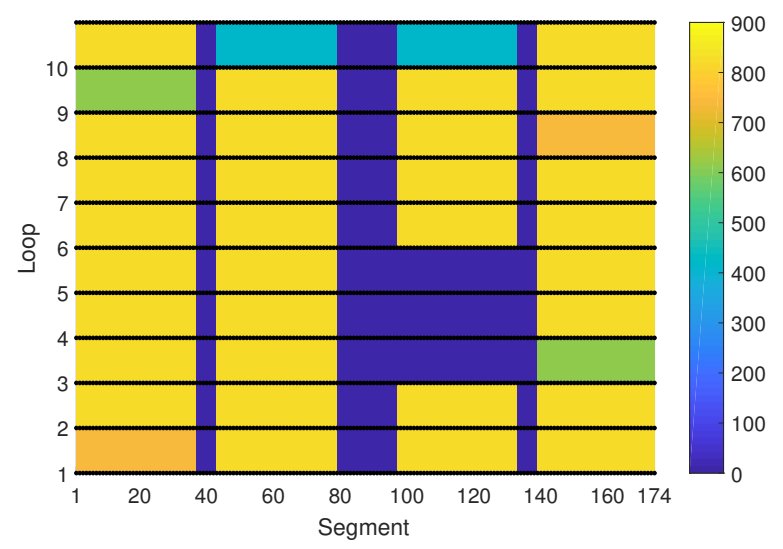

(a) With no clouds

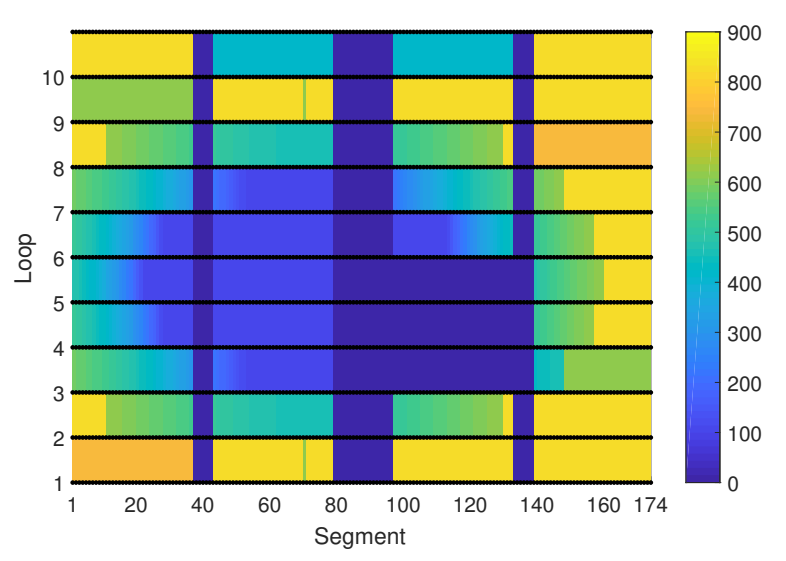

(b) With a cloud at $t=70 \mathrm{~min}$.

Figure 2: Effective solar DNI profile on the 10-loop collector field. 


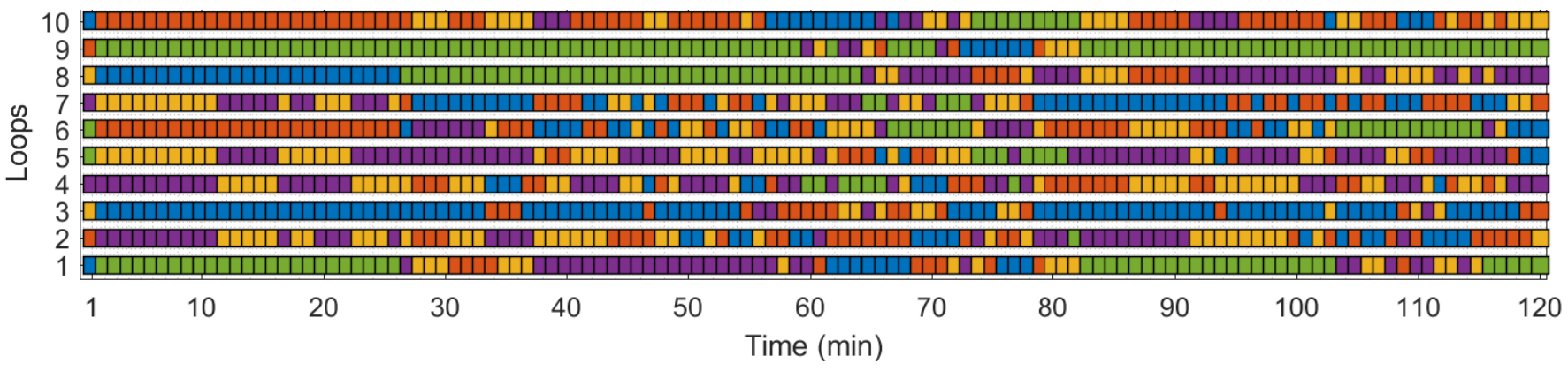

Figure 3: Formation of coalitions in the 10-loop plant with the coalitional MPC method, where loops in a coalition are given by the same color.

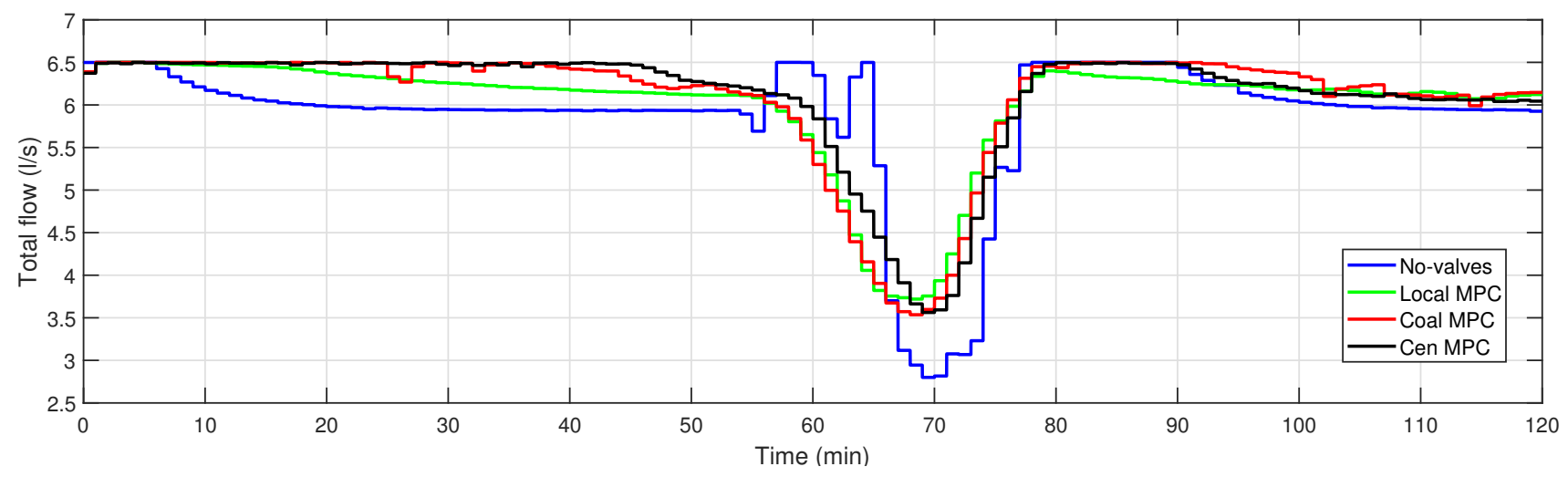

Figure 4: Total flow for the 10-loop collector field.

The simulation length of the ACUREX plant is $N_{\text {sim }}=$ $120 \mathrm{~min}$, and the two-hour effective solar DNI $\left(\eta_{i, j}^{\mathrm{col}} G_{i, j} I_{i, j}(k)\right)$ profile used as disturbances is shown in Fig. 2. Even if there were no clouds (Fig. 2 (a)), it is considered that there are some dusty and defocused segments, which have a lower effective solar DNI (e.g., the first thirty-six segments of loops \#1 and \#9). Additionally, it is considered a moving cloud entering at loop \#10 from $t=63 \mathrm{~min}$ to $t=76 \mathrm{~min}$. As observed in Fig. 2(b), the effective solar DNI at $t=70 \mathrm{~min}$ decreases significantly. This moving cloud simulate small cumulus of clouds that affect solar collector fields on partially cloudy days.

Fig. 3 depicts the formation of clusters of loops in the coalitional MPC scheme, where the y-axis indicates each loop and the $\mathrm{x}$-axis the simulation time. Since pairs of unbalanced loops are associated, five colors represent the five coalitions at that time instan ${ }^{3}$ For example, the partition of the system is $\mathcal{P}=\{\{1,9\},\{2,4\},\{3,8\},\{5,7\},\{6,10\}\}$ at time instant $t=10 \mathrm{~min}$. The total flow rate resulting from applying the four MPC schemes in the ACUREX plant is shown in Fig. 4 For instance, the flow rate and the outlet temperature of the clean loop \#2 and the dusty loop \#3 are also represented in Fig. 5. Observe that the moving cloud, which appears between $t=63 \mathrm{~min}$ and $t=76 \mathrm{~min}$, causes a decrease of the flow rate to hold a suitable temperature and maximize the thermal power.

In Table 3, the mean thermal powers obtained in the ACUREX plant (10-loop plant) applying the four control strate-

\footnotetext{
${ }^{3}$ The reader is referred to the online version of the article to interpret the color legends of most figures.
}

Table 3: Numerical results for the proposed controllers in the 10-loop plant

\begin{tabular}{cccc}
\hline Method & Mean power & Improvement & $\boldsymbol{\tau}_{C}$ \\
\hline No-valves & $1118.6 \mathrm{~kW}$ & - & $26.98 \mathrm{~s}$ \\
Local MPC & $1126.6 \mathrm{~kW}$ & $0.72 \%$ & $0.44 \mathrm{~s}$ \\
Coal MPC & $1130.0 \mathrm{~kW}$ & $1.01 \%$ & $3.28 \mathrm{~s}$ \\
Cen MPC & $1131.3 \mathrm{~kW}$ & $1.09 \%$ & $240.3 \mathrm{~s}$ \\
\hline
\end{tabular}

gies are displayed. As expected, centralized MPC provides the best performance by obtaining a $1.09 \%$ of improvement with regard to the no-valves method. Conversely, the high computation time of centralized control $\tau_{C}=240.3 \mathrm{~s}$ for the 10-loop plant will make impractical its real-time implementation in a larger size plant since the considered sample time of controllers in solar plants is around $\Delta t_{\mathrm{ctr}}=60 \mathrm{~s}$. In this sense, decentralized and coalitional strategies are more suitable to control large-scale systems. Improvements of $0.72 \%$ and $1.01 \%$ are attained with Local MPC and Coal MPC, respectively.

Concerning the 100-loop plant, it is used a simulation length of $N_{\text {sim }}=100 \mathrm{~min}$ and the two-hour effective solar DNI $\left(\eta_{i, j}^{\mathrm{col}} G_{i, j} I_{i, j}(k)\right)$ profiles that are represented in Fig. 6. Profile $\mathrm{A}$ is characterized by three small moving clouds that partially cover the plant between $t=35 \mathrm{~min}$ and $t=76 \mathrm{~min}$. In Fig.6(a), it is represented the solar DNI profile A, where two of three clouds appear. Conversely, the profile B presents a large moving cloud that almost covers the 100-loop plant, as shown in Fig. 6(b). Moreover, figs. 7 and 8 depict, respectively, the total 

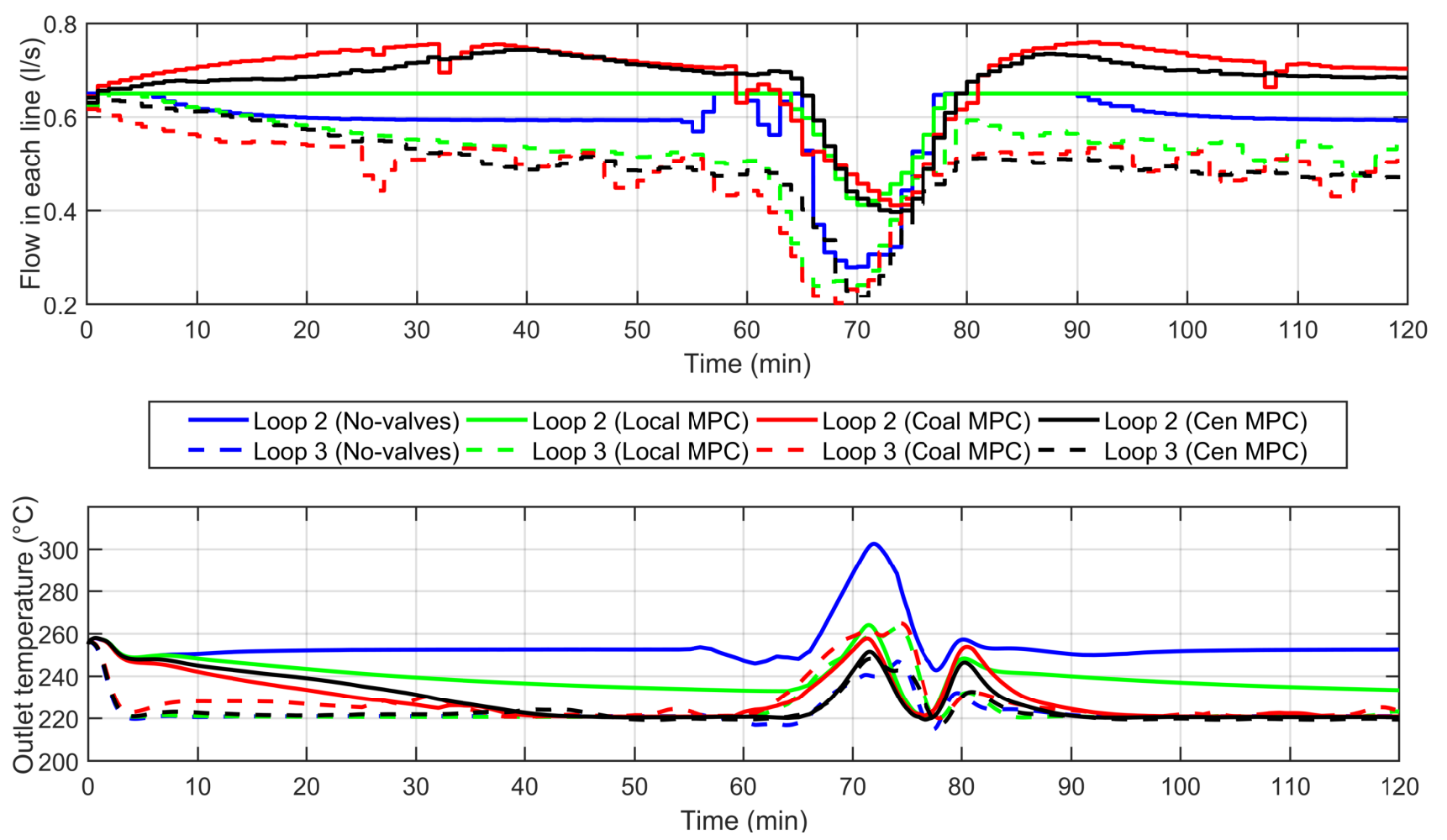

Figure 5: Flow and outlet temperature for two loops (a dusty loop, and a clean loop) of the 10-loop plant

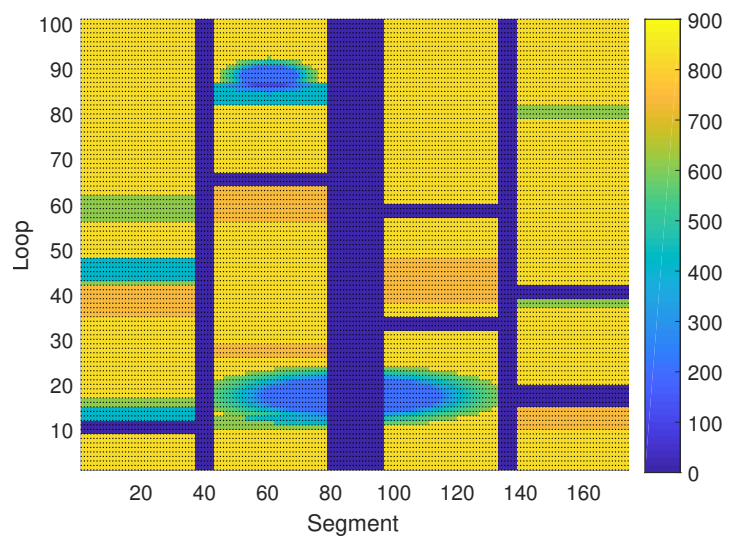

(a) Profile A

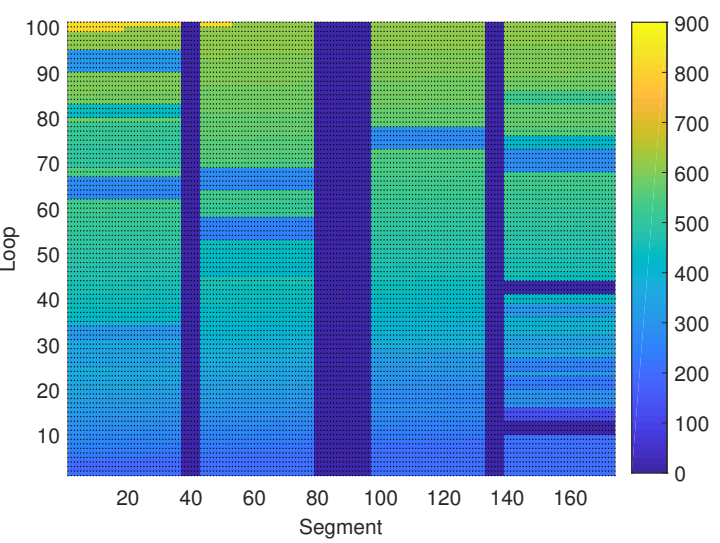

(b) Profile B

Figure 6: Effective solar DNI profile at $t=70 \mathrm{~min}$ for the 100-loop collector field.

Table 4: Numerical results for the proposed methods in the 100-loop plant

\begin{tabular}{c|ccc|ccc}
\hline & \multicolumn{3}{|c}{ Profile A } & \multicolumn{3}{c}{ Profile B } \\
Method & Mean power & Impr. & $\tau_{C}$ & Mean power & Impr. & $\tau_{C}$ \\
\hline No-valves & $11647 \mathrm{~kW}$ & - & $202.8 \mathrm{~s}$ & $9379.4 \mathrm{~kW}$ & - & $249.7 \mathrm{~s}$ \\
Local MPC & $11702 \mathrm{~kW}$ & $0.45 \%$ & $0.31 \mathrm{~s}$ & $9460.6 \mathrm{~kW}$ & $0.87 \%$ & $0.42 \mathrm{~s}$ \\
Coal MPC & $11744 \mathrm{~kW}$ & $0.79 \%$ & $2.86 \mathrm{~s}$ & $9482.8 \mathrm{~kW}$ & $1.10 \%$ & $3.14 \mathrm{~s}$ \\
Cen MPC & $11763 \mathrm{~kW}$ & $1.00 \%$ & $130.07 \mathrm{~min}$ & $9512.9 \mathrm{~kW}$ & $1.42 \%$ & $150.22 \mathrm{~min}$ \\
\hline
\end{tabular}

flow, and the flow and outlet temperature for two loops using the solar DNI profile A. Likewise, the results obtained by im- plementing the four control methods to the 100-loop plant with the solar DNI profile B are represented in figs. 9 and 10 Ob- 


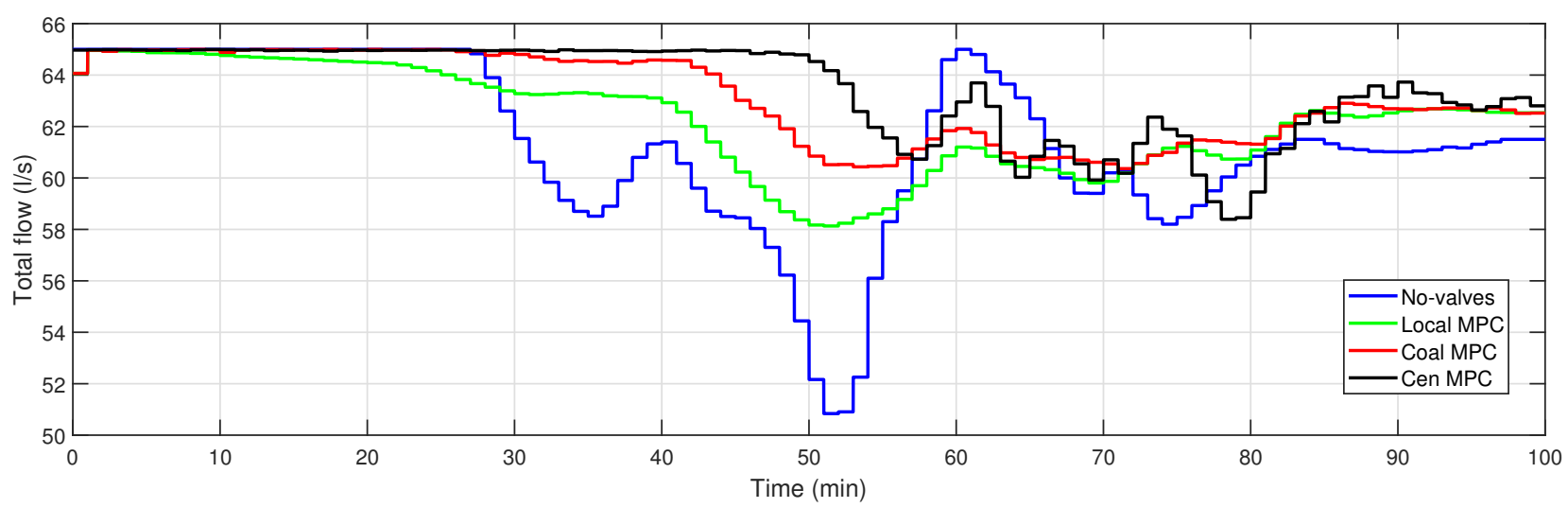

Figure 7: Total flow for the 100-loop plant with DNI profile A.

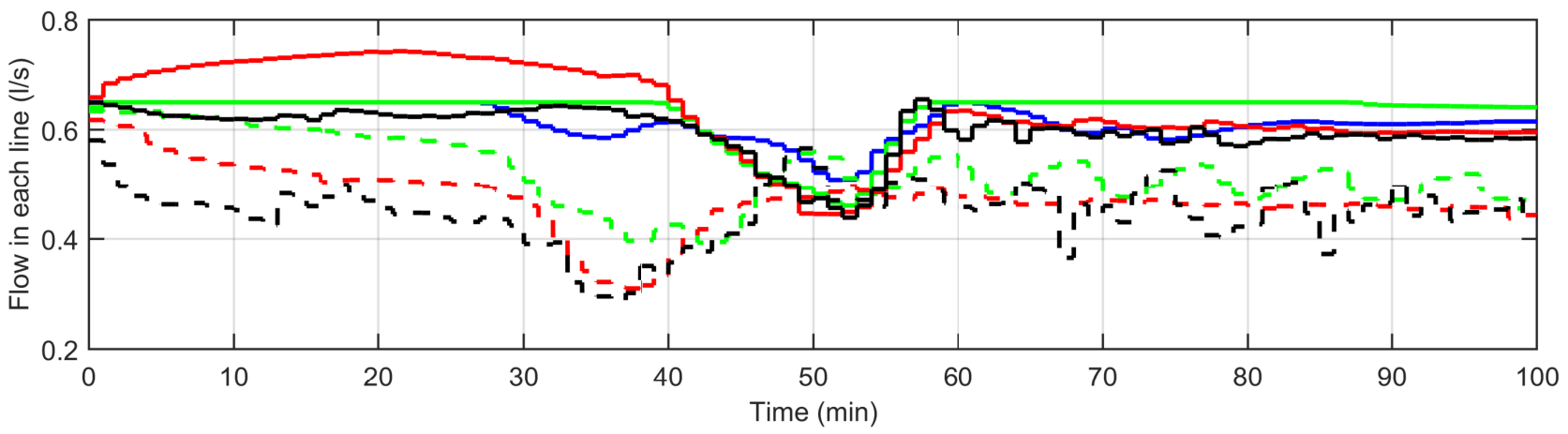

- Loop 43 (No-valves) - Loop 43 (Local MPC) - Loop 43 (Coal MPC) - Loop 43 (Cen MPC)

- - Loop 57 (No-valves) - - Loop 57 (Local MPC) - - Loop 57 (Coal MPC) - - Loop 57 (Cen MPC)

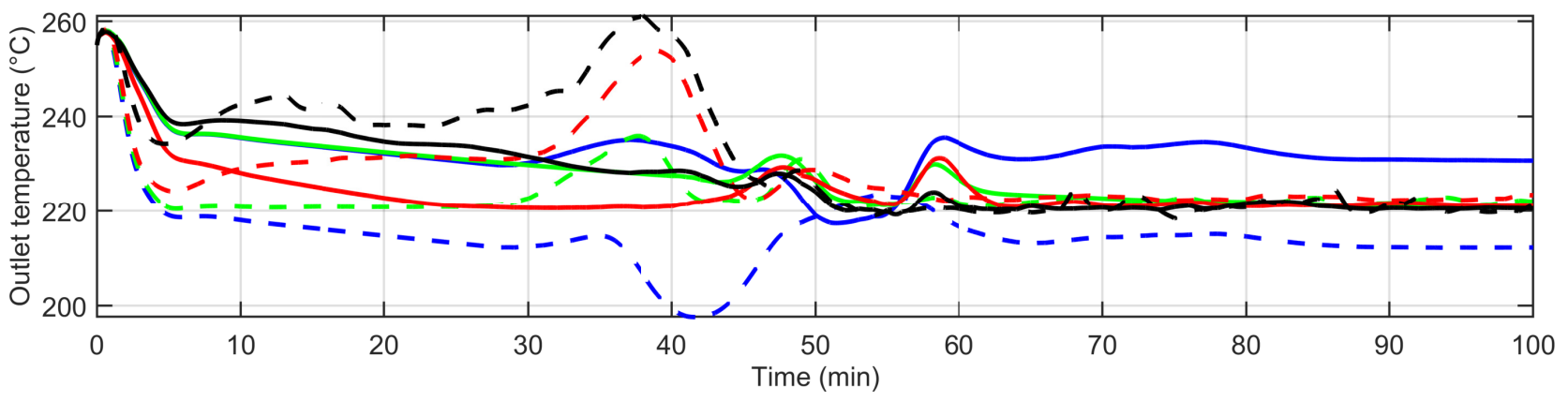

Figure 8: Flow and outlet temperature for two loops (a dusty loop and a clean loop) of the 100-loop plant with DNI profile A.

serve the decrease in flow rate to increase the temperature when clouds partially or fully cover the loops. In absence of clouds, local controllers $C=\{i\}$ generally try to reduce outlet temperatures to maximize thermal power. However, the temperatures of loops with the lowest effective solar DNI could not reach the minimum $T^{\mathrm{f} \text {,min }}=220^{\circ} \mathrm{C}$ due to the maximum flow per loop $(0.65 \mathrm{l} / \mathrm{s})$, which is the constraint considered by Local MPC.

On the other hand, using centralized and coalitional MPC, the outlet temperatures of these loops reach lower values and, thus, it is entailed a larger thermal power than using Local MPC. This gain is achieved by increasing the flow of the saturated loops using the margin provided by other loops with lower effective solar DNI. For example, as seen in Fig. 8, coalitional MPC increases the flow in loop \#43 by using the margin pro- vided by loop \#57, while the total flow is saturated on its maximum value $q_{\mathrm{T}}^{\max }=65 \mathrm{l} / \mathrm{s}$ in the time interval from $t=0$ to $t=30 \mathrm{~min}$, approximately.

Table 4 displays the mean thermal power obtained in the 100loop field with both solar DNI profiles. As for the no-valve method in the 100-loop plant, it is outperformed by Local MPC a $0.45 \%$ and by coalitional MPC a $0.79 \%$ when they are implemented with the DNI profile A. Similarly, it is obtained an improvement of $0.87 \%$ and $1.10 \%$ with Local MPC and Coal MPC, respectively, when DNI profile B is applied. In both cases, centralized MPC provides the best performance at the expense of substantial computation times $\left(\tau_{C}=130.07 \mathrm{~min}\right.$ with profile $\mathrm{A}$, and $\tau_{C}=150.22$ min with profile $\mathrm{B}$ ), which are not feasible to control distributed solar collector fields of larger 


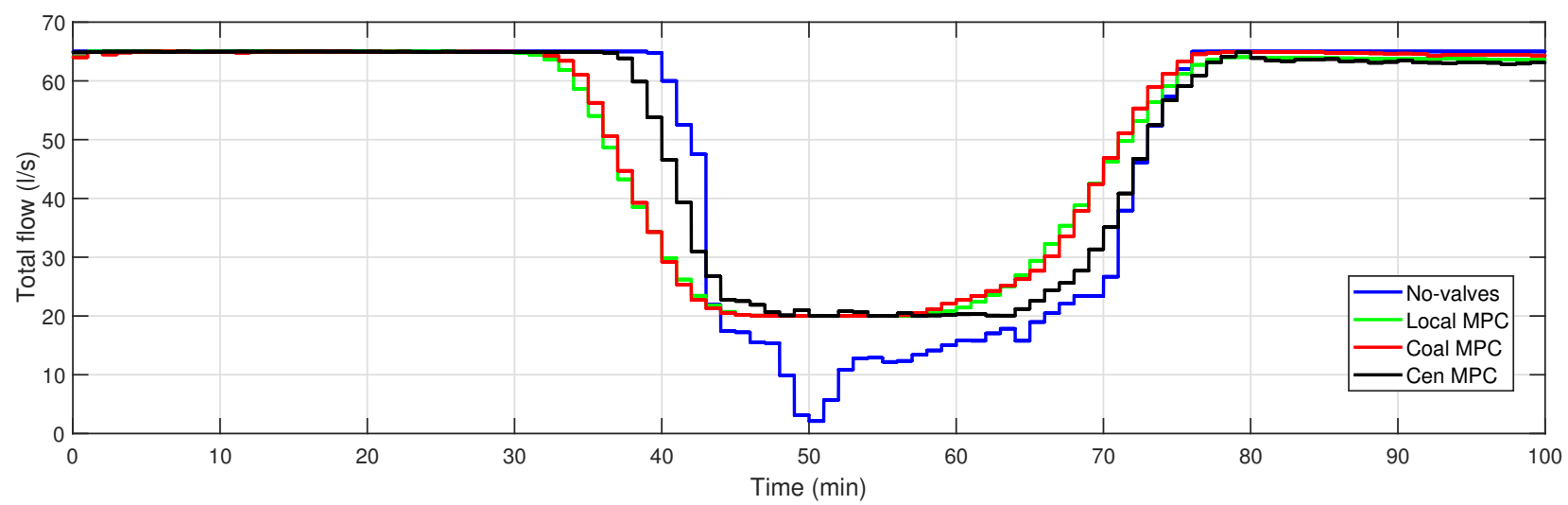

Figure 9: Total flow for the 100-loop plant with DNI profile B.
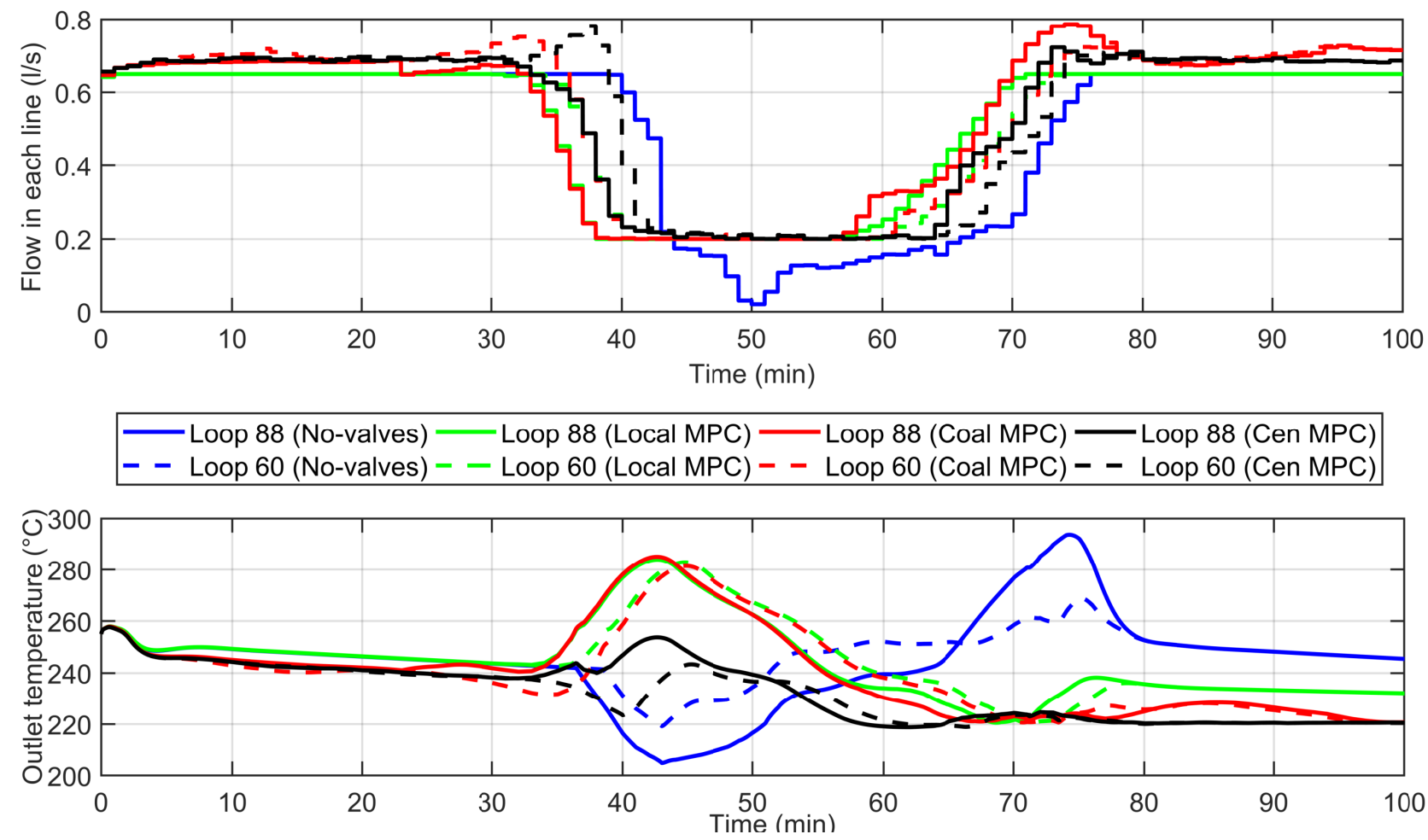

Figure 10: Flow and outlet temperature for two loops of the 100-loop plant with DNI profile B.

size in real-time.

Remark 5. Although improvements of less than $1.5 \%$ in all MPC methods can appear not to be very high, it is economically profitable at the cost of only implementing the proposed algorithm if the valves are already in place in the solar parabolictrough plants.

\subsection{Discussion: Clustering criteria}

This section discusses two particular key issues related with the clustering criteria. The first one is the number of potential partitions, and the second is the size of coalitions.

i) The number of system partitions exponentially increases with the set of possible cooperation links $\mathcal{L}$, i.e., $2^{|\mathcal{L}|}$. If a exhaustive search algorithm were used, $2^{5}$ and $2^{50}$ partitions in the 10-loop plant $(\mathcal{L}=5)$ and the 100-loop plant ( $\mathcal{L}=50$ ) should be considered, respectively. This combinatorial explosion makes unfeasible to find the partition of the system $\mathcal{P}(k)$ in real-time for large-scale systems. Hence, there is a need for employing clustering criteria with a variety of heuristics to reduce the number of possible partitions. The criterion considered in Algorithm 1 to define the partition $\mathcal{P}(k)$ is to associate pairs of loops with high gaps of flow rates to balance the plant. Thus, a loop of coalition $C$ can exploit the extra flow rate of the other loop belonging to $C$. This fact increases the resulting thermal energy of the solar parabolic-trough plants when the field is unbalanced. Other versions of the proposed coalitional MPC algorithm have been tested in the ACUREX 
plant. For example, it is obtained a $0.89 \%$ and a $0.93 \%$ of improvement when clustering pairs of loops according to significant differences in the previous average temperature and the final temperature, respectively. A higher performance $(1 \%)$ is obtained by associating pair of loops with high gaps of solar DNI over the prediction horizon. However, we obtain lower improvements than $1.01 \%$, which is attained with the current criterion.

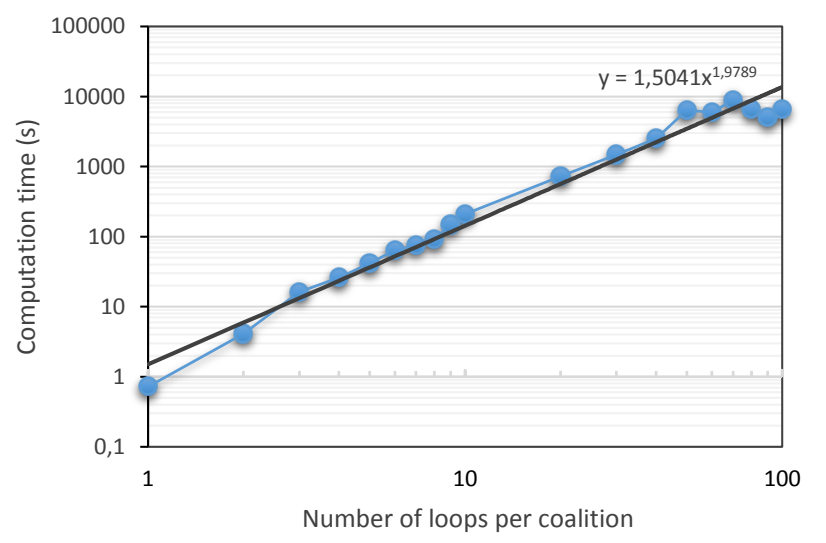

Figure 11: Computation time according to the coalition size.

ii) Another issue is the coalition size, which is closely related with the performance and the computation time. As an example, it is performed a simulation of $N_{\text {sim }}=10 \mathrm{~min}$ to illustrate this fact. In Fig. 11, the logarithmic scaled vertical axis displays the computation time in seconds, and the horizontal axis displays the coalition size. The trend line becomes $y=1.5041 x^{1.9789}$, which approximates the obtained data points using least squares. Since the computation time grows with the square of the coalition size, it is justified the need of downsizing the number of loops per coalition. Indeed, only clusters of two loops are allowed in Algorithm 1 to find a trade-off between performance and computation burden. Greater size of coalitions could be allowed if faster mechanisms such as neurodynamic optimizations would be employed to speed up the computation in real-time. This topic will be studied in future work.

\section{Conclusions}

A hierarchical coalitional MPC algorithm for maximizing the thermal power of large-scale solar parabolic-trough plants has been proposed. The supervisory controller decides the partition of the plant dynamically in such a way that the resulting thermal power is maximized, and local controllers adopt that cooperative structure. The proposed approach clusters pairs of unbalanced loops to benefit from the extra flow rates in those loops of the coalition with low solar DNI and efficiency due to dust or maintenance.

The method proposed was assessed in two simulated solar parabolic-trough plants. The first one is the ACUREX plant, which was located in Almería (Spain) and had ten loops, and the second one is a 100-loop extension of ACUREX. In the simulations, synthetic solar DNI profiles with moving clouds partially covering the plants were employed with some dusty and defocused segments to add realism.

Simulation results show the improvements in the thermal power obtained with the proposed coalitional MPC algorithm compared to no-valves and decentralized MPC. Although the best performance is obtained with centralized MPC, its considerable computation time makes its implementation unfeasible in real-time. Hence, the proposed coalitional MPC becomes a suitable strategy to control large-scale solar collector fields properly.

Future research will focus on using a detailed hydraulic model of the system, a fully distributed implementation of the proposed strategy, and the use of neural networks to reach a consensus on the optimal control actions.

\section{Acknowledgements}

The authors gratefully acknowledge the financial support by the European Research Council [Advanced Grant OCONTSOLAR number 789051], the Spanish Ministry of Economy [project number DPI2017-86918-R], and the Spanish Ministry of Science, Innovation, and Universities [grant numbers FPU18/04476 and IJC2018-035395-I].

\section{References}

Ahandani, M.A., Kharrati, H., Hashemzadeh, F., Baradarannia, M., 2020. Decentralized switched model-based predictive control for distributed largescale systems with topology switching. Nonlinear Analysis: Hybrid Systems 38, 100912.

Alonso-Montesinos, J., Batlles, F.J., Portillo, C., 2015. Solar irradiance forecasting at one-minute intervals for different sky conditions using sky camera images. Energy Conversion and Management 105, 1166-1177.

Alsharkawi, A., Rossiter, J.A., 2016. Dual mode MPC for a concentrated solar thermal power plant. IFAC-PapersOnLine 49, 260-265.

Andrade, G.A., Pagano, D.J., Alvarez, J.D., Berenguel, M., 2013. A practical NMPC with robustness of stability applied to distributed solar power plants. Solar Energy 92, 106-122.

Barreiro-Gomez, J., Ocampo-Martinez, C., Quijano, N., 2019. Time-varying partitioning for predictive control design: Density-games approach. Journal of Process Control 75, 1-14.

Berenguel, M., Cirre, C.M., Klempous, R., Maciejewski, H., Nikodem, M., Nikodem, J., Rudas, I., Valenzuela, L., 2005. Hierarchical control of a distributed solar collector field, in: International Conference on Computer Aided Systems Theory, Springer. pp. 614-620.

Camacho, E.F., Berenguel, M., Rubio, F.R., Martínez, D., 2012. Solar energy fundamentals, in: Collection of Control of Solar Energy Systems. Springer, pp. $1-23$.

Camacho, E.F., Gallego, A.J., 2013. Optimal operation in solar trough plants: A case study. Solar Energy 95, 106-117.

Camacho, E.F., Gallego, A.J., 2015. Model predictive control in solar trough plants: a review. IFAC-PapersOnLine 48, 278-285.

Camacho, E.F., Rubio, F.R., Berenguel, M., Valenzuela, L., 2007a. A survey on control schemes for distributed solar collector fields. part i: Modeling and basic control approaches. Solar Energy 81, 1240-1251.

Camacho, E.F., Rubio, F.R., Berenguel, M., Valenzuela, L., 2007b. A survey on control schemes for distributed solar collector fields. part ii: Advanced control approaches. Solar Energy 81, 1252-1272.

Chanfreut, P., Maestre, J.M., Camacho, E.F., 2020. Coalitional model predictive control on freeways traffic networks. IEEE Transactions on Intelligent Transportation Systems , 1-12. 
Chu, Y., Pedro, H.T.C., Coimbra, C.F.M., 2013. Hybrid intra-hour dni forecasts with sky image processing enhanced by stochastic learning. Solar Energy 98, 592-603.

Cirre, C.M., Berenguel, M., Valenzuela, L., Klempous, R., 2009. Reference governor optimization and control of a distributed solar collector field. European Journal of Operational Research 193, 709-717.

Devabhaktuni, V., Alam, M., Depuru, S.S.S.R., Green II, R.C., Nims, D., Near, C., 2013. Solar energy: Trends and enabling technologies. Renewable and Sustainable Energy Reviews 19, 555-564.

Ding, B., Ge, L., Pan, H., Wang, P., 2016. Distributed MPC for tracking and formation of homogeneous multi-agent system with time-varying communication topology. Asian Journal of Control 18, 1030-1041.

Dörfler, F., Jovanović, M.R., Chertkov, M., Bullo, F., 2014. Sparsity-promoting optimal wide-area control of power networks. IEEE Transactions on Power Systems 29, 2281-2291.

Fele, F., Debada, E., Maestre, J.M., Camacho, E.F., 2018. Coalitional control for self-organizing agents. IEEE Transactions on Automatic Control 63, 2883-2897.

Fele, F., Maestre, J.M., Camacho, E.F., 2017. Coalitional control: Cooperative game theory and control. IEEE Control Systems Magazine 37, 53-69.

Fele, F., Maestre, J.M., Hashemy, S.M., de la Peña, D.M., Camacho, E.F., 2014 Coalitional model predictive control of an irrigation canal. Journal of Process Control 24, 314-325.

Frejo, J.R.D., Camacho, E.F., 2020. Centralized and distributed model predictive control for the maximization of the thermal power of solar parabolictrough plants. Solar Energy 204, 190-199.

Gallego, A.J., Camacho, E.F., 2012. Adaptative state-space model predictive control of a parabolic-trough field. Control Engineering Practice 20, 904 911.

Gálvez-Carrillo, M., De Keyser, R., Ionescu, C., 2009. Nonlinear predictive control with dead-time compensator: Application to a solar power plant. Solar Energy 83, 743-752.

Gao, J., Yang, X., Liu, D., 2017. Uncertain shapley value of coalitional game with application to supply chain alliance. Applied Soft Computing 56, 551556.

Johansen, T.A., Storaa, C., 2002. Energy-based control of a distributed solar collector field. Automatica 38, 1191-1199.

La Bella, A., Bonassi, F., Farina, M., Scattolini, R., 2019. Two-layer model predictive control of systems with independent dynamics and shared control resources. IFAC-PapersOnLine 52, 96-101.

Law, E.W., Prasad, A.A., Kay, M., Taylor, R.A., 2014. Direct normal irradiance forecasting and its application to concentrated solar thermal output forecasting-a review. Solar Energy 108, 287-307.

Maestre, J.M., Ishii, H., 2017. A pagerank based coalitional control scheme. International Journal of Control, Automation and Systems 15, 1983-1990.

Maestre, J.M., Negenborn, R.R., 2014. Distributed model predictive control made easy. volume 69. Springer.

Masero, E., Fletscher, L.A., Maestre, J.M., 2020. A coalitional model predictive control approach for heterogeneous cellular networks, in: Proceedings of the European Control Conference (ECC), IEEE. pp. 448-453.

Montes, M.J., Rubbia, C., Abbas, R., Martínez-Val, J.M., 2014. A comparative analysis of configurations of linear fresnel collectors for concentrating solar power. Energy 73, 192-203.

Navas, S.J., Rubio, F.R., Ollero, P., Lemos, J.M., 2018. Optimal control applied to distributed solar collector fields with partial radiation. Solar Energy 159, 811-819.

Niyato, D., Wang, P., Saad, W., Hjorungnes, A., 2011. Controlled coalitional games for cooperative mobile social networks. IEEE Transactions on Vehicular Technology 60, 1812-1824.

Ramirez, L., Vindel, J.M., 2017. Forecasting and nowcasting of dni for concentrating solar thermal systems, in: Collection of Advances in Concentrating Solar Thermal Research and Technology. Elsevier, pp. 293-310.

Rossiter, J.A., 2003. Model-based predictive control: a practical approach. CRC press.

Salomé, A., Chhel, F., Flamant, G., Ferrière, A., Thiery, F., 2013. Control of the flux distribution on a solar tower receiver using an optimized aiming point strategy: Application to themis solar tower. Solar Energy 94, 352-366.

Sánchez, A.J., Gallego, A.J., Escaño, J., Camacho, E., 2018. Temperature homogenization of a solar trough field for performance improvement. Solar Energy 165, 1-9.

Santos-Martin, D., Alonso-Martinez, J., Eloy-Garcia, J., Arnalte, S., 2012. So- lar dish-stirling system optimisation with a doubly fed induction generator. IET Renewable Power Generation 6, 276-288.

Schiffer, J., Dörfler, F., Fridman, E., 2017. Robustness of distributed averaging control in power systems: Time delays $\&$ dynamic communication topology. Automatica 80, 261-271.

Silva, R.N., Lemos, J.M., Rato, L.M., 2003. Variable sampling adaptive control of a distributed collector solar field. IEEE Transactions on Control Systems Technology 11, 765-772.

Tang, W., Allman, A., Pourkargar, D.B., Daoutidis, P., 2018. Optimal decomposition for distributed optimization in nonlinear model predictive control through community detection. Computers \& Chemical Engineering 111, 43-54.

Torrico, B.C., Roca, L., Normey-Rico, J.E., Guzman, J.L., Yebra, L., 2010. Robust nonlinear predictive control applied to a solar collector field in a solar desalination plant. IEEE Transactions on Control Systems Technology 18, 1430-1439.

Twidell, J., Weir, T., 2015. Renewable energy resources. Routledge.

Yuan, L., Meng, K., Dong, Z.Y., 2018. Hierarchical control scheme for coordinated reactive power regulation in clustered wind farms. IET Renewable Power Generation 12, 1119-1126.

Zhang, H.L., Baeyens, J., Degrève, J., Cacères, G., 2013. Concentrated solar power plants: Review and design methodology. Renewable and Sustainable Energy Reviews 22, 466-481. 